\title{
Long chain imidazolium ionic liquids as templates in the formation of mesoporous silica nanospheres
}

\begin{abstract}
Mesoporous silica material was first discovered by Mobil research group in 1990s. Its nanoscale form is favorable due to their properties such as high specific surface area, tunable sizes and easy surface functionalization. Mesoporous silica nanoparticles (MSNs) is commonly synthesize through condensation of silica source in the presence of templates. Two most commonly used templates are cetyltrimethylammonium bromide (CTAB) and polymer PF-127. However, in these recent years, new templates were discovered and one of it is ionic liquids (ILs). ILs are salt that has melting point below $100{ }^{\circ} \mathrm{C}$ and its core structure is similar to CTAB which consist of large organic cations and inorganic or organic anions. Therefore, it has the potential to serve as alternative template in formation of MSNs. This work reported usage of two long chain imidazolium ILs as template in the synthesis of monodispersed mesoporous silica nanospheres (MNSs). The effect of imidazolium ILs alkyl chain length on properties of MNSs were investigated. It was found that particle size of MNSs decreases with alkyl chain length of imidazolium ILs. The porosity of the MNSs were further analyzed through nitrogen sorption analysis where the surface areas were 570.61 and $598.71 \mathrm{~m} 2 \mathrm{~g}-1$ and pore volume up to $1.4 \mathrm{~cm} 3$ g-1.
\end{abstract}

Keyword: Imidazolium ionic liquids, Ionic liquids, Mesoporous silica nanoparticles 